\title{
A Large Biaxial Shear Box for Shaking Table Test on Saturated Sand
}

\begin{abstract}
A large flexible laminar shear box was developed for the study of the behavior of saturated sand, especially liquefaction, and soil-structure interactions under two-dimensional earthquake shaking on a shaking table at the National Center for Research on Earthquake Engineering (NCREE) in Taiwan. The shear box is composed of 15 layers of aluminum alloy inner and outer frames with a specimen size of 1880 by 1880 by $1520 \mathrm{~mm}$. The soil at various depths inside the shear box can move, without torsion, according to the two-dimensional wave action induced by the shaking table. The sand specimen inside the shear box is prepared by the wet sedimentation method from a large pluviation device. Instruments are installed to measure the displacements, accelerations, and water pressures at various locations. Shaking table tests of the laminar shear box with and without a sand specimen were conducted. The test results showed that the performance of the biaxial laminar box and the instrumentation are satisfactory.
\end{abstract}

KEYWORDS: earthquake, shaking table, laminar box, two-dimensional shaking, liquefaction, sand, specimen preparation

\section{Introduction}

The soil in a level ground of an infinite extent under earthquake shaking is usually modeled as a soil element undergoing a simple shear loading condition. In order to study the soil behaviors, such as strain-stress relationship and liquefaction, small soil specimens were tested in the laboratory using triaxial compression apparatus, simple shear devices, and torsional shear apparatus under regular or irregular dynamic loads. In these types of tests, the stress conditions and deformations in the soil elements are significantly affected by the boundary conditions, and the loading conditions are generally not the true field situations due to the limitations of the loading devices and the size of specimens. Therefore, large soil specimens have been placed on shaking tables that can reproduce the actual seismic ground shaking according to the earthquake recording under either $1 \mathrm{~g}$ or centrifugal conditions (e.g., De Alba et al. 1976; Hushmand et al. 1988; Taylor et al. 1995). Thus, the soil behaviors under the more realistic seismic loading conditions can be observed and analyzed.

At present, most studies of seismic soil behaviors, such as liquefaction, are tested and modeled under one-dimensional shaking, i.e., the loading and soil responses, such as displacements and accelerations, are in the direction of one axis in the horizontal plane. The understanding of the soil behaviors under two- and threedimensional seismic loading conditions is very limited. For the two-dimensional shaking discussed in this paper, the loading and

Manuscript received May 17, 2004; accepted for publication June 2, 2005; published November 2005.

${ }^{1}$ Professor, Department of Civil Engineering, National Taiwan University, Taipei 106, Taiwan.

${ }^{2}$ Section Chief, Civil Engineering Section, Public Work Bureau, Ilan County Government, Ilan 263, Taiwan.

${ }^{3}$ Postdoctoral Researcher, Center for Bridge Engineering Research, National Central University, Jhongli 320, Taiwan.

${ }^{4}$ Research Associate, National Center for Research on Earthquake Engineering, Taipei 106, Taiwan.

${ }^{5}$ Former graduate student, Department of Civil Engineering, National Taiwan University, Taipei 106, Taiwan. soil responses can be in any direction in the horizontal plane of two ( $\mathrm{X}$ and $\mathrm{Y}$ ) axes, and they also change with time. This becomes a multidirectional problem. Pyke et al. (1975) studied the settlement of dry sand under two-dimensional shaking by testing a 76-mm layer of dry sand on a shaking table. They found that the volumetric strain of sands under two-dimensional loading is equal to the sum of the individual volumetric strain in a one-dimensional test. Ishihara and Yamazaki (1980) and Ishihara and Nagase (1988) conducted multidirectional simple shear tests on saturated sand specimens of $70 \mathrm{~mm}$ in diameter and $30 \mathrm{~mm}$ in height. The results showed that the liquefaction resistance in specimens under multidirectional loading with equal amplitudes in two perpendicular directions decreased to at most $60 \%$ of the liquefaction resistance under one-directional loading of the same cyclic stress ratio. In the case of actual irregular seismic loading, the liquefaction resistance reduced to about $85 \%$ of that in unidirectional loading. Kammerer et al. (2002) performed multidirectional simple shear tests on a medium to high relative density, saturated sand, $150 \mathrm{~mm}$ in diameter, and $19 \mathrm{~mm}$ in height in a bidirectional simple shear device. They found that the shear strains resulting from the multidirectional loading can be much larger than those estimated based on one-dimensional testing; however, larger shear stresses do not always lead to larger strains.

Until recently, only very limited results were reported by Endo and Komanobe (1995) on a multidirectional shaking table test of a large sand specimen (1200 $\mathrm{mm}$ in diameter, $1400 \mathrm{~mm}$ in height) using a laminar cylindrical shear container. They found that the sand exhibited less dilatancy and can liquefy easier under the circular rotational shaking than under one-directional shaking. The performance of the shear container and the motion responses induced by the two-dimensional shaking were not discussed. No other large-scale physical model test under two-dimensional loading, either on a $1 \mathrm{~g}$ shaking table or in a centrifuge, was reported. Therefore, for a better understanding and to provide the data for verification of the analyses and modeling of soil responses under two-dimensional earthquake loading, a large-scale biaxial shear box on a shaking table has been developed at the National Center for Research on Earthquake Engineering (NCREE) in Taiwan to 


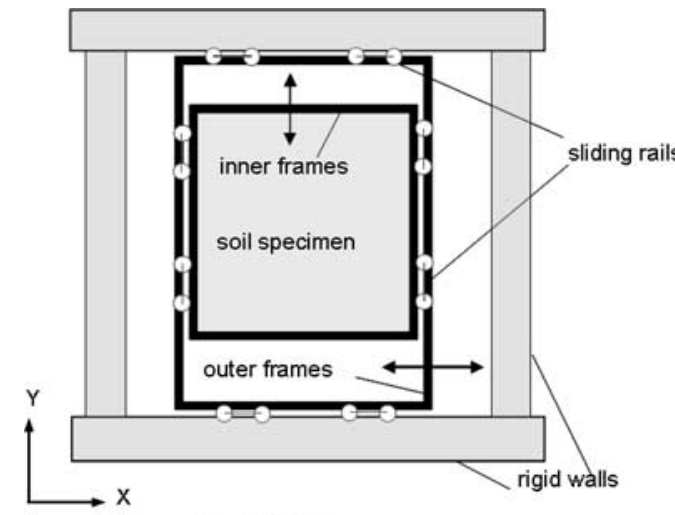

(a) plan view

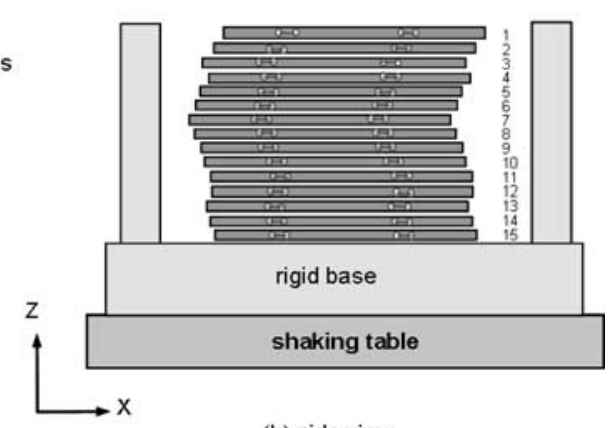

(b) side view

FIG. 1-Schematic drawings of the biaxial laminar shear box.

simulate the in situ multidirectional earthquake shaking on soils.

\section{Biaxial Laminar Shear Box}

\section{Design Considerations}

A soil layer under a level ground surface is usually in a $K_{0}$ condition, while, during an earthquake, the soil at different depths may move differently in the horizontal plane following the upward shear wave propagation. To provide such flexible but unyielding side boundaries as in the field, laminar simple shear boxes composed of layers of frames are commonly used in the tests. For horizontal twodimensional earthquake shaking, every layer of the frames should be able to move freely in every direction, i.e., multidirectionally, in a horizontal $(\mathrm{X}-\mathrm{Y})$ plane that follows the movement of the soil in the container. This can be accomplished if the frames are allowed to move biaxially in both $\mathrm{X}$ and $\mathrm{Y}$ axes simultaneously.

To allow biaxial motions, the laminar shear box requires a special mechanism design. Firstly, the center of mass of every layer of soil and frame is unlikely at the geometrical center of the horizontal cross section. Secondly, the centers of mass of different layers will not be aligned on the same vertical line, particularly when there are shear deformations. Therefore, the inertia force due to shaking will then produce some torque on every layer of soil about a vertical

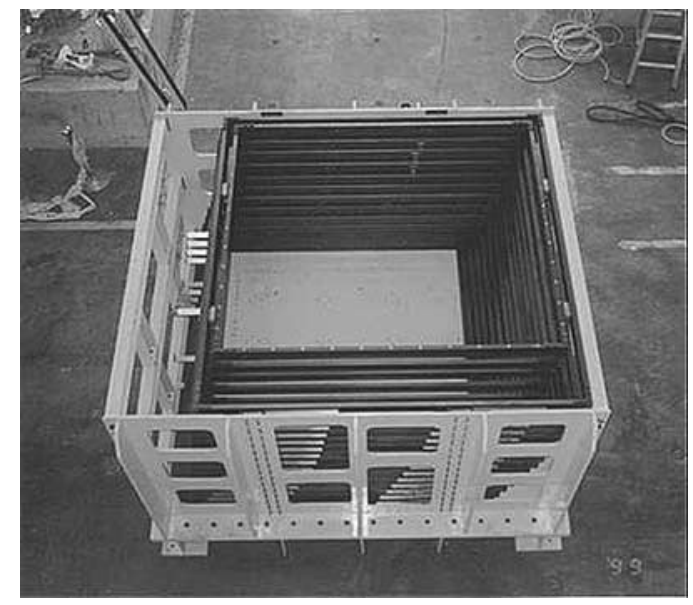

FIG. 2-Picture of the empty laminar shear box. axis due to the eccentricity of the mass center. Without adequate restraints, this would introduce torsional motions of the soil, which in turn cause different shear stress and strain conditions at different locations on the same horizontal plane. The interpretation and analysis of the test results thus become very complicated and inaccurate. Therefore, not only should the shear box provide free movements in multiple directions but also it must ensure non-torsional motion of the soil specimen during shaking.

\section{Assembly of the Laminar Box}

The laminar shear box developed at NCREE is composed of 15 layers of sliding frames as schematically shown in Fig. 1. Each layer consists of two nested frames, an inner frame with inside dimensions of 1880 by $1880 \mathrm{~mm}$ and an outer frame with inside dimensions of $1940 \mathrm{~mm}$ by $2340 \mathrm{~mm}$. Both frames are made of a special aluminum alloy (specific gravity $=2.70$ ) with $30 \mathrm{~mm}$ in thickness and $80 \mathrm{~mm}$ in height, except the uppermost layer which
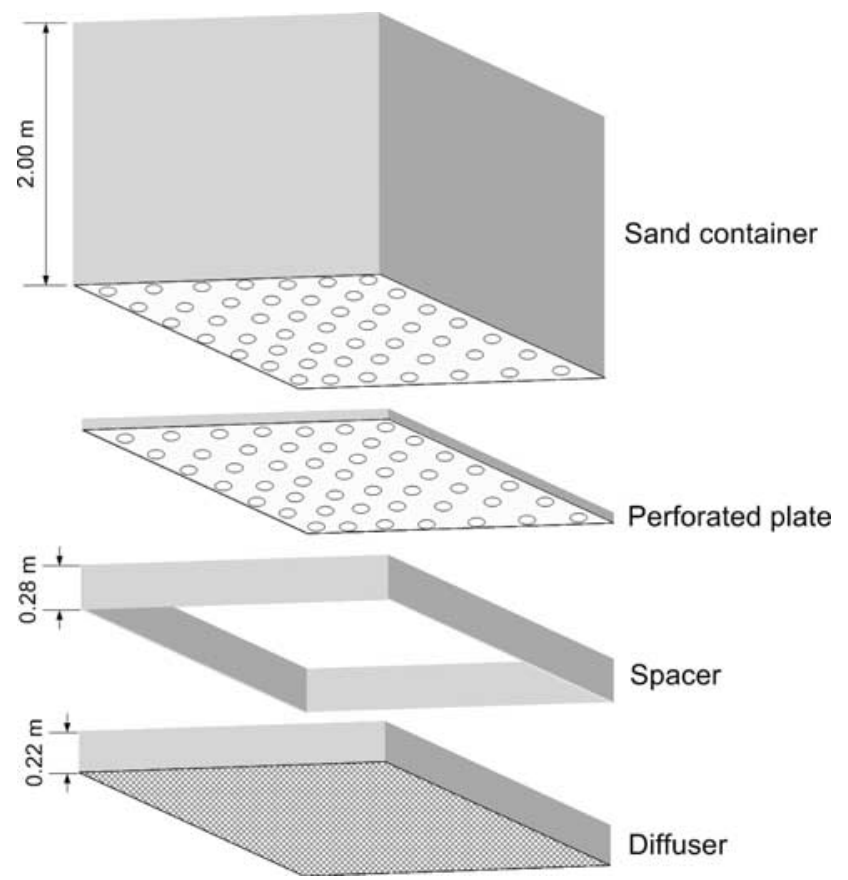

FIG. 3-Pluviator for specimen preparation. 


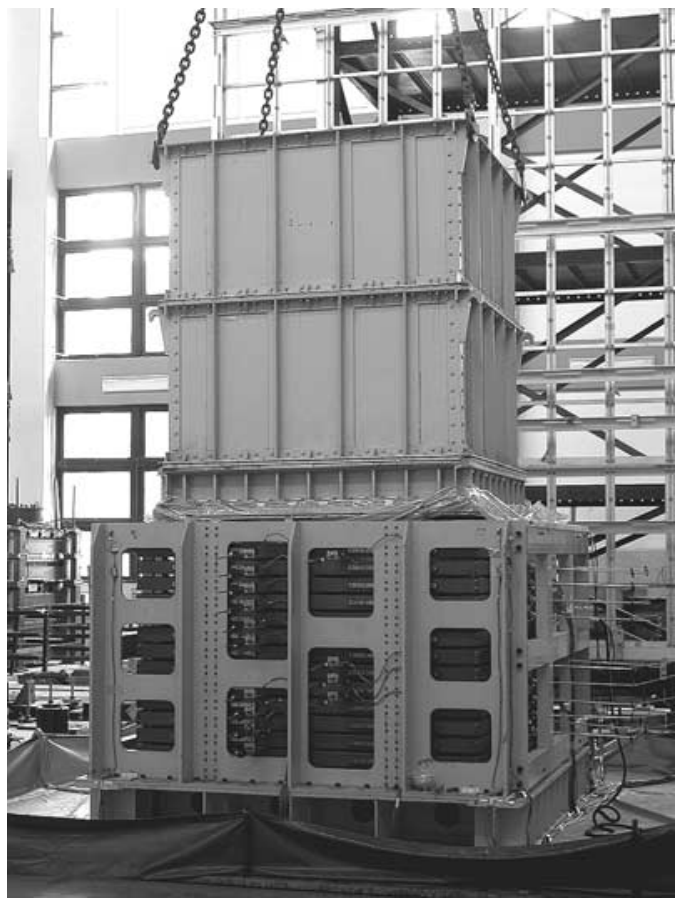

FIG. 4-Specimen preparation with the pluviator.

has a height of $100 \mathrm{~mm}$. The aluminum alloy is adopted for its sufficient strength and rigidity to provide unyielding boundaries, and for its light weight to minimize the effect of inertia of the frame on the soil movements. These 15 layers of frames are separately supported on the surrounding rigid steel walls, one above the other, with a vertical gap of $20 \mathrm{~mm}$ between the adjacent layers. The 20 -mm gap is provided to avoid rupture of the rubber membrane inside the box during a large relative deformation between layers. This gap was considered narrow enough to prevent the membrane from excessive bulging and the sideward expansion of soil. Thus, a sand specimen of $1880 \mathrm{~mm}$ by $1880 \mathrm{~mm}$ by $1520 \mathrm{~mm}$ can be placed inside the inner frames. The mass of each layer of frames is about $14 \%$ of the mass of a $100-\mathrm{mm}$ layer of soil enclosed by the inner frame.

Linear guideways consisting of sliding rails and bearing blocks are used to allow an almost frictionless horizontal movement without vertical motions. Each outer frame is supported by the sliding rails mounted on two opposite sides of the outer rigid walls. The

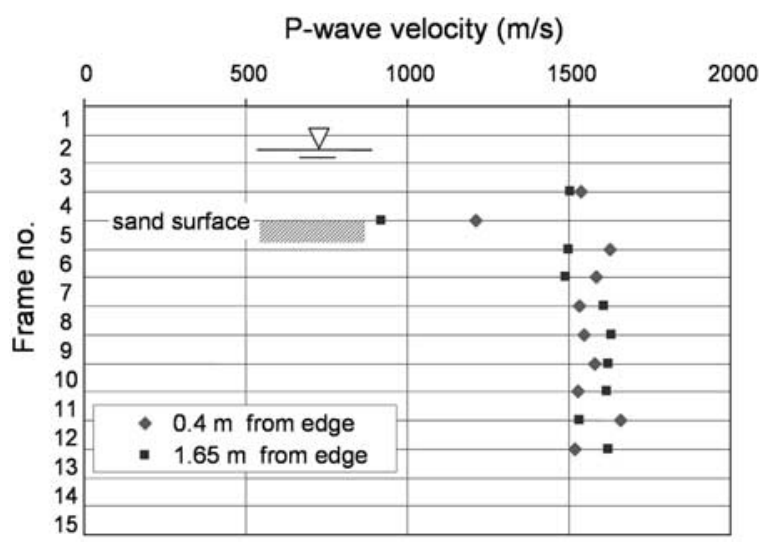

FIG. 5-P-wave velocities of the sand specimen after preparation.
TABLE 1-Properties of Vietnam sand.

\begin{tabular}{cccccc}
\hline Shape & $G_{\mathrm{s}}$ & $D_{50}(\mathrm{~mm})$ & $C_{\mathrm{u}}$ & $e_{\max }$ & $e_{\min }$ \\
\hline Subangular & 2.65 & 0.30 & 1.72 & 0.912 & 0.610 \\
\hline
\end{tabular}

bearing blocks on the outer frame allow its movement in the $\mathrm{X}$ direction with minimal friction. Similarly, sliding rails are also provided for each outer frame to support the inner frame of the same layer so that the inner frame can move in the $\mathrm{Y}$ direction with respect to the outer frame, which is moving in the $\mathrm{X}$ direction at the same time. With these 15 nested layers of inner and outer frames supported independently on the rigid walls, the soil at each depth can move in a multidirectional fashion in the horizontal plane without torsion in response to the wave actions induced by the shaking table. The key advantage of this design is that the movement of each layer of the frames is independent of that of other layers of frames at different depths. The effects of friction and inertia of the frames will not accumulate along the depth of the laminar box and large relative motion between layers of different depths is possible. A maximum displacement of $\pm 150 \mathrm{~mm}$ is allowed for each layer in both $\mathrm{X}$ and $\mathrm{Y}$ directions. The final design and manufacturing were completed in cooperation with Mechanical Industry Research Laboratories at Industrial Technology Research Institute, Hsinchu, Taiwan. Figure 2 shows a picture of the completed laminar shear box.

\section{Provisions for a Watertight Box}

A 2-mm-thick silicone rubber membrane was placed inside the box to provide a watertight shear box to contain the saturated soil. The silicone rubber membrane was fixed at the top and bottom of the shear box. A silicone sealant was applied at the seams to prevent

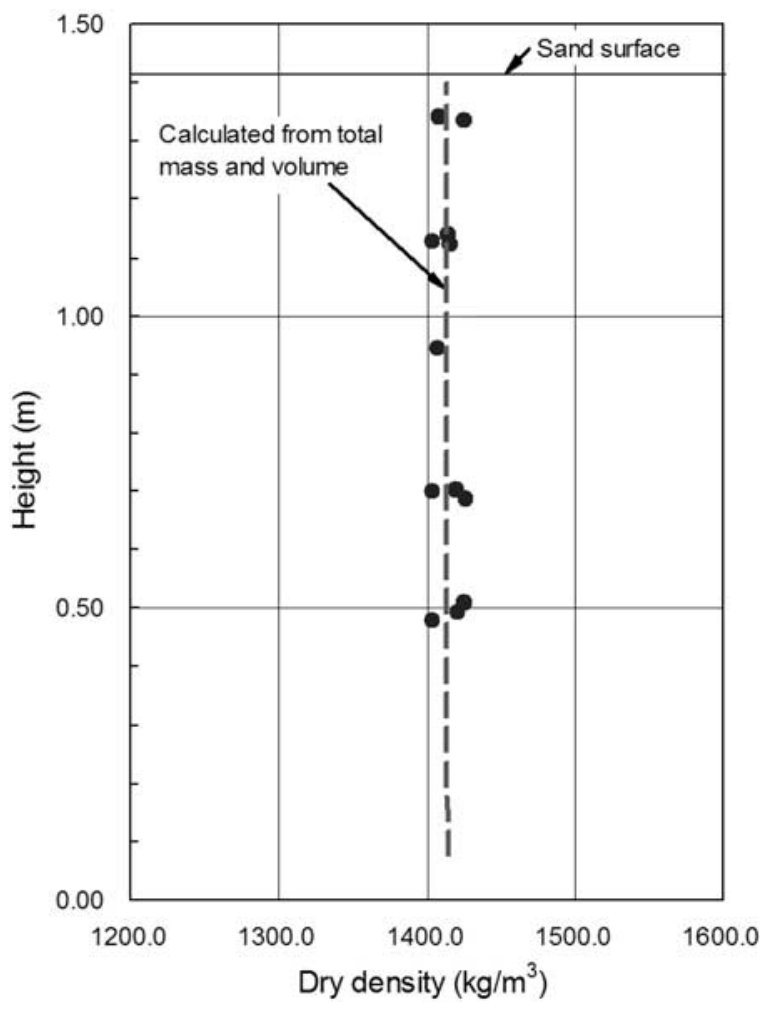

FIG. 6-Density of the sand specimen after preparation. 


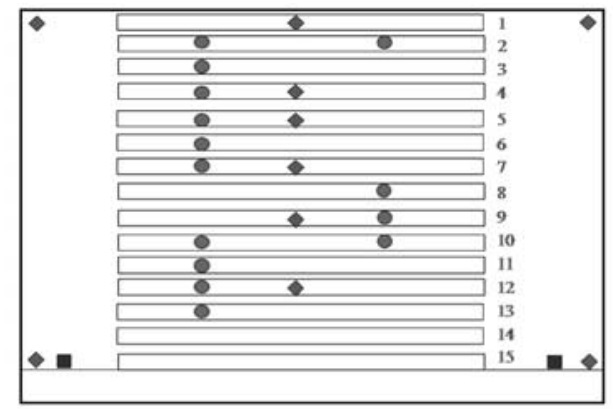

(a) Inner frame

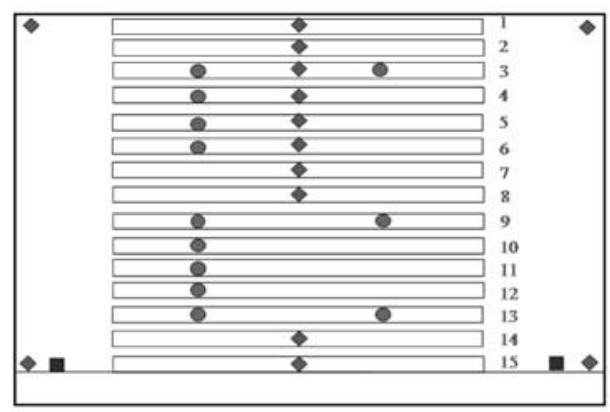

(b) Outer frame

- LDT velocity transducer $\bullet$ accelerometer

FIG. 7-Locations of instrumentation on the frames.

leakage. An approximately 50-mm-thick layer of pea gravel is placed at the bottom of the box with a layer of geotextile at the top and bottom of the gravel. There are sixteen 50-mm-diameter holes for the intake and drainage of water.

\section{Sand Specimen Preparation}

Preparation methods for a large dry sand specimen, such as the raining method, to control its uniformity and density have been re-

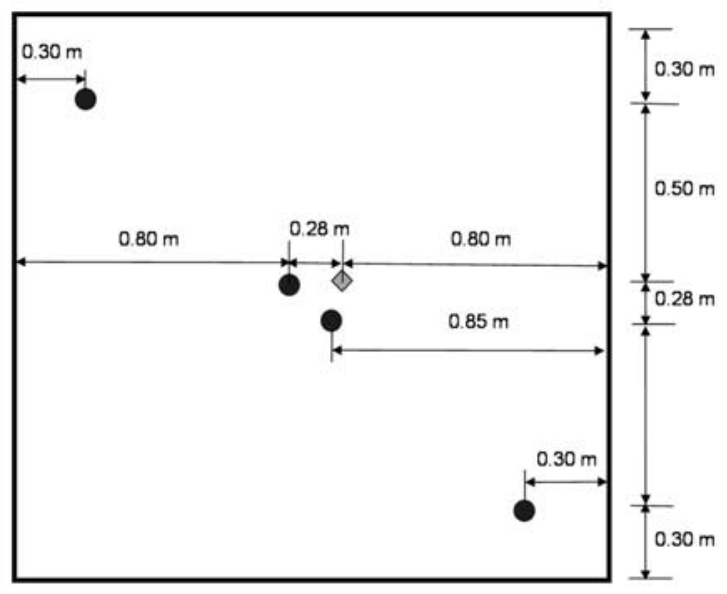

(a) plan view

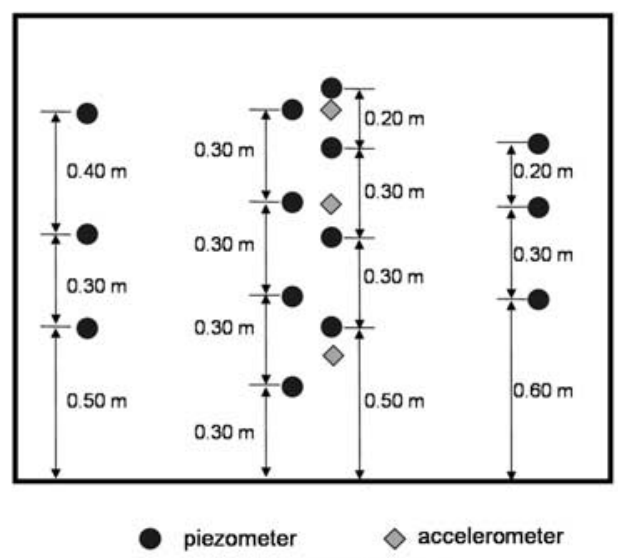

(b) vertical view

FIG. 8-Locations of piezometers and accelerometers inside the specimen. ported by many researchers, e.g., Rad and Tumay (1985) and Fretti et al. (1995). However, very little information is available for preparing large saturated specimens to obtain a desired uniformity, density, and saturation. In this study, a special pluviator was designed for preparing the sand specimen inside the shear box by the raining method. The pluviator, as shown in Fig. 3, is composed of the following:

- A container for the needed volume of sand ( $\approx 7 \mathrm{Mg}$ in mass) to fill the shear box. The bottom of the container is perforated with 40-mm-diameter holes, $140 \mathrm{~mm}$ center to center.

- A motor-driven perforated plate, which is exchangeable for various hole diameters ranging from 15 to $40 \mathrm{~mm}$ to control the rate of pluviation and in turn the density of the specimen. At first, the container is shut with dislocated hole positions between the movable plate and the bottom of the container. The plate is then moved to match the hole locations to let the sand rain down through the holes.

- A diffuser comprised of four layers of the No. 4 sieves with a spacing of $70 \mathrm{~mm}$ to obtain a uniform deposit over the cross section of the shear box during pluviation. The direction of

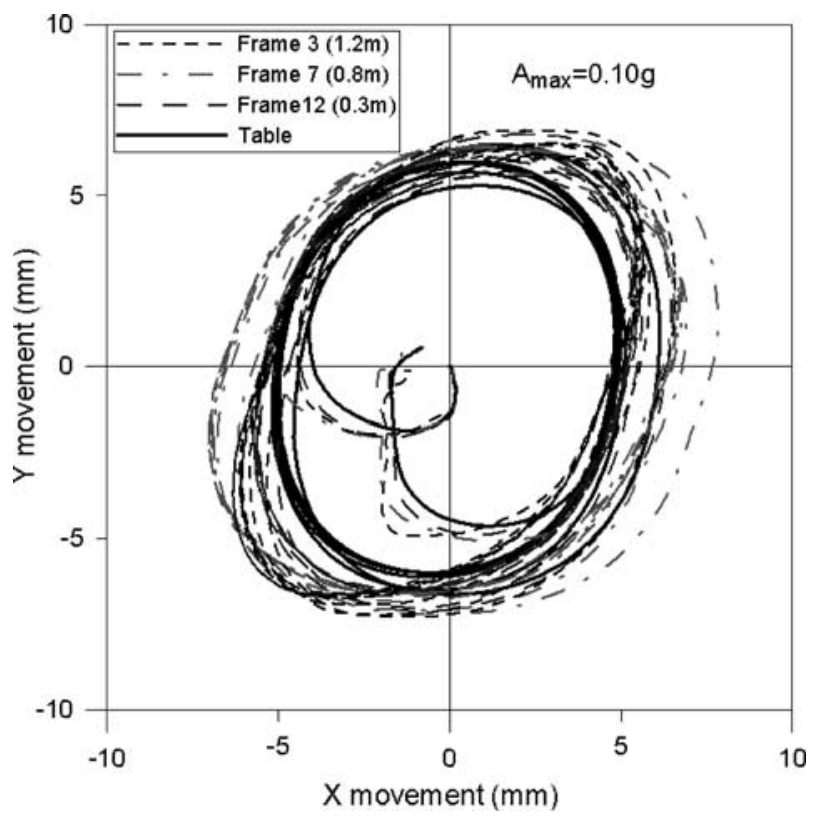

FIG. 9-Frame movements in the horizontal plane under two-dimensional shaking. 


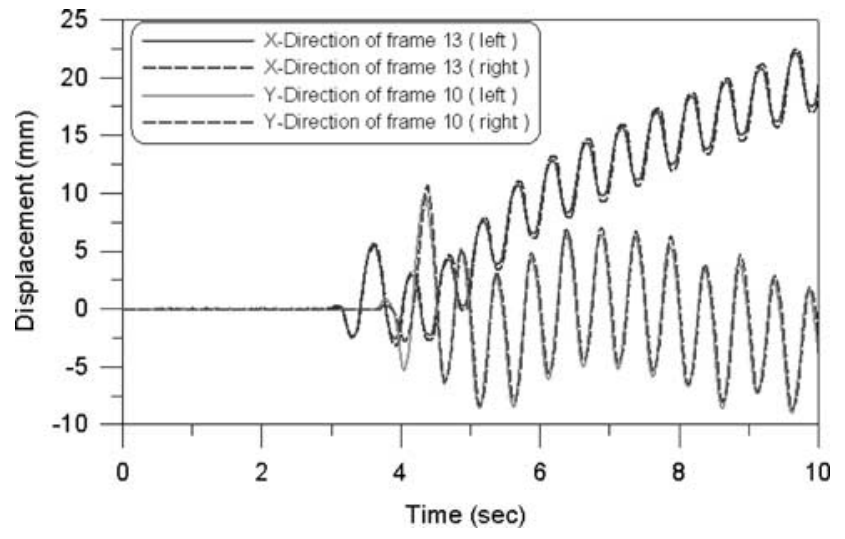

FIG. 10-Displacements at two different locations on frames 10 and 13 during a shaking test.

the sieve wires of each layer changes by $45^{\circ}$ from that of the next layer.

Commercially available fine silica sand from Vietnam was used in this study for the convenience of obtaining a large quantity and good gradation control of the sand. The basic properties of this sand are given in Table 1. It was found that saturation of a specimen by introducing water into the dry sand was very difficult and unreliable according to an experiment using a smaller glass container ( 500 by 230 by $350 \mathrm{~mm}$ ) under an acrylic pluviator ( 500 by 230 by $350 \mathrm{~mm}$ ) with a similar perforated plate and diffuser. The wet sedimentation method was thus adopted for the specimen preparation in this study. The dry sand was rained down into the laminar box filled with water to a precalculated depth as shown in Fig. 4. The saturation of the specimen prepared by this method was verified by measuring the P-wave velocity across the specimen horizontally at different depths. A small steel ball hit the sand specimen at the 20-mm gaps between the frames and the arrival times of the P-wave were measured using the accelerometers close to the hitting point and across the specimen. Figure 5 shows the results of P-wave velocity measurements at two vertical sections of the sand specimen. It can be seen that the sand was well saturated with P-wave velocities between 1500 and $1700 \mathrm{~m} / \mathrm{s}$ at various depths (including the water above the sand surface) but not at the sand surface, probably

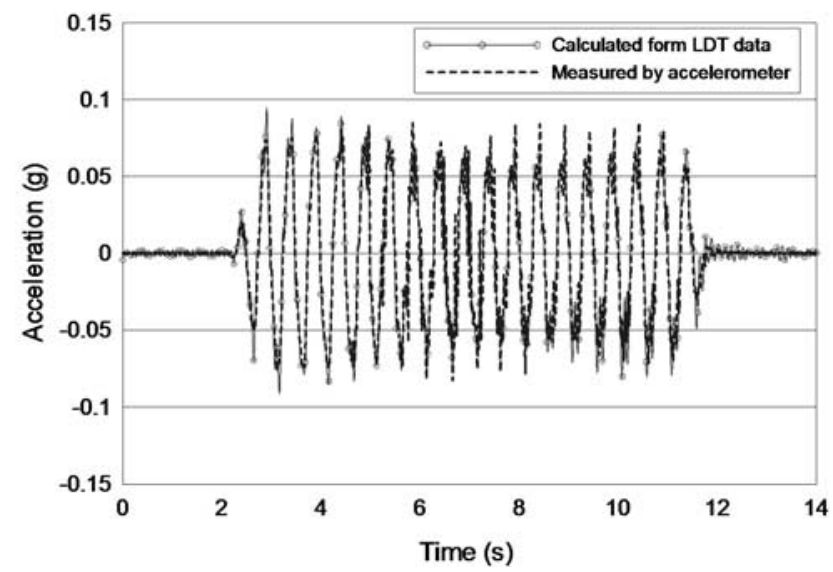

FIG. 11-Comparison of frame motions measured by an accelerometer and $L D T$.

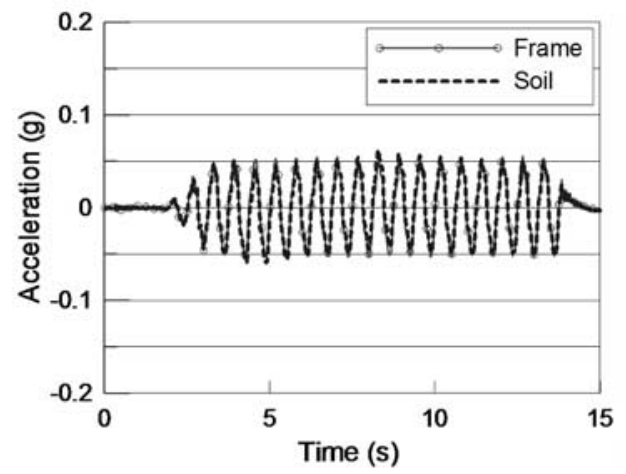

(a) Acceleration time history

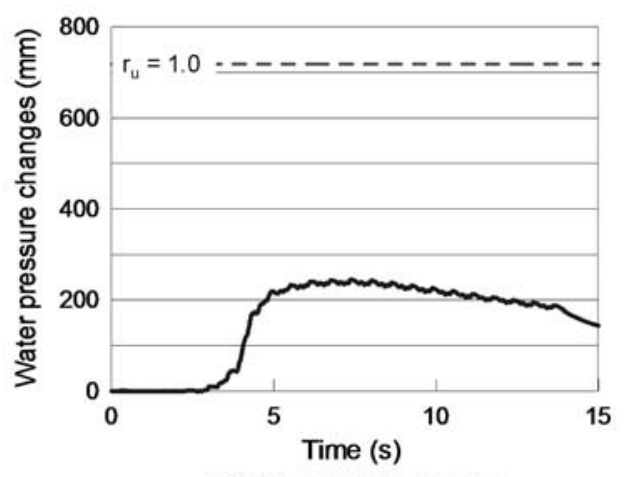

(b) Water pressure changes

FIG. 12-Measured acceleration and pore pressure changes at $0.80 \mathrm{~m}$ below the sand surface under a shaking of $A_{\max }=0.05 \mathrm{~g}$, January 2003.

because of minor unevenness of the surface and traces of air bubbles.

Cone penetration tests (CPT) using a miniature cone with a diameter of $8.9 \mathrm{~mm}$ at different locations were attempted to check the uniformity and density. However, correlation between the tip resistance and the sand density was not established owing to the insufficiency of test data and erratic results for very loose sand at shallow depths of low overburden pressures. No meaningful CPT result was obtained for a sand specimen right after pluviation. Further evaluations are underway of the use of the miniature CPT on the denser sand specimen after shaking tests.

In order to directly measure the density and uniformity of large sand specimens, short thin-walled tube samples $(\approx 150 \mathrm{~mm}$ in length and $73 \mathrm{~mm}$ in diameter) were taken at various locations and depths inside the shear box. These thin-walled tubes were pushed into the sand at various elevations during excavation of the sand from the shear box, and the samples were taken by carefully removing the surrounding soil. Figure 6 shows the densities of the sand specimen at different depths immediately after specimen preparation. It can be seen that uniformity of the sand density was very good by the preparation method used in this study.

The densities of the sand are approximately 1410 and $1490 \mathrm{~kg} / \mathrm{m}^{3}$ for the specimens prepared using the plate hole sizes of 15 and $40 \mathrm{~mm}$, respectively. The plate with 15 -mm-diameter holes was used the most in this study. During the shaking table tests, the density of the sand specimen increased after each shaking as discussed later in this paper. 


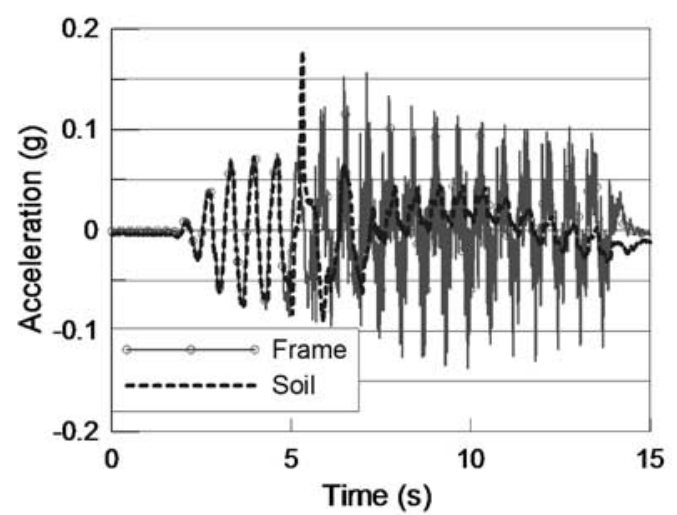

(a) Acceleration time history

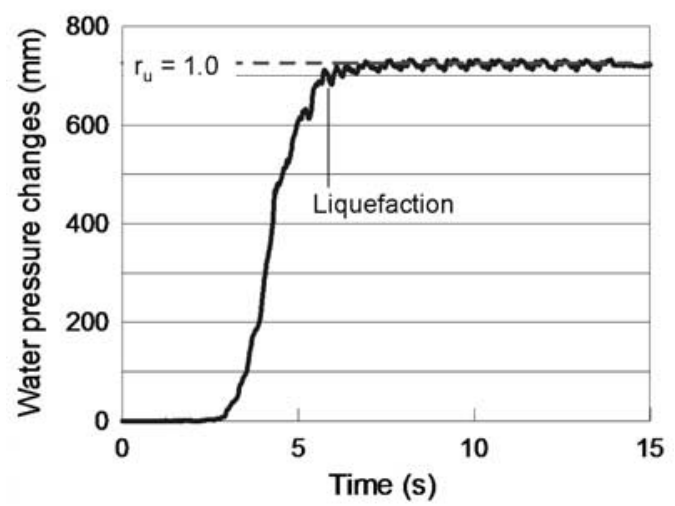

(b) Water pressure changes

FIG. 13-Measured acceleration and pore pressure changes at $0.80 \mathrm{~m}$ below the sand surface under a shaking of $A_{\max }=0.075 \mathrm{~g}$, January 2003.

\section{Instrumentation}

\section{On the Frames of the Laminar Box}

Transducers for displacement and acceleration measurements were placed at various locations and depths on the outside rigid walls, the outer frames for X-direction motions, and the inner frames for Y-direction motions. Displacement of the frames can be measured using magnetostriction-type linear displacement transducers (LDT) even when there are some movements perpendicular to the direction of the measuring displacements. Four velocity transducers are also placed on the outside walls to measure the velocities of the box for comparison purposes. Figure 7 shows the layout of the instrumentation on the shear box.

\section{Within the Soil Specimen}

Miniature piezometers $(6.4 \mathrm{~mm}$ in diameter and $11.4 \mathrm{~mm}$ in length) with a ceramic filter are installed inside the box for pore water pressure measurements at different locations and depths. In addition, small-sized piezoresistive accelerometers of $7.9 \mathrm{~mm}$ (hex) by $10.9 \mathrm{~mm}$ (length) are also placed for measuring the accelerations at various locations within the soil in both $\mathrm{X}$ and $\mathrm{Y}$ directions. These transducers are positioned with thin fishing lines before placing the sand into the shear box. These fishing lines were cut prior to the shaking tests. Preliminary measurements are taken and checked for the piezometers at different depths below the water

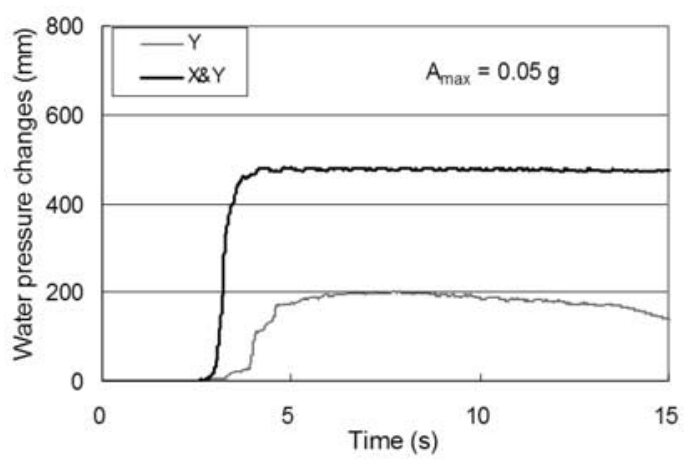

(a) $659 \mathrm{~mm}$ below sand surface

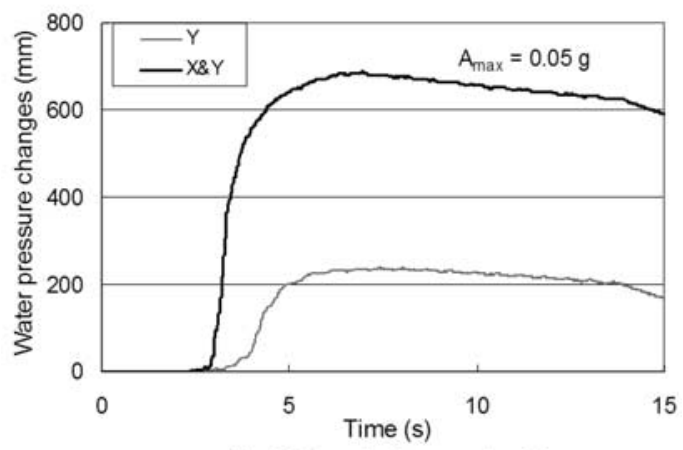

(b) $1053 \mathrm{~mm}$ below sand surface

FIG. 14-Pore water pressure changes during 1-D and 2-D shaking, January 2003.

surface after they are underwater for at least 3 days to ensure the proper functioning of these transducers. Figure 8 shows the locations of the piezometers and accelerometers within the soil specimen. It should be noted that these sensors would settle along with the sand inside the box under shaking. Thus the positions of the sensors were checked prior to every shaking test by water pressure readings, which in turn were converted to the depths below the water surface.

\section{Shaking Table Tests}

After completion of the shear box, the empty box, with and without filling of water, was placed on the shaking table at NCREE and test runs were conducted under both one- and two-dimensional shakings (1-D and 2-D, respectively). The force required to overcome the static friction before the movement of the frames ranges from 40 to $120 \mathrm{~N}$, which is relatively small compared to the weight of a $100-\mathrm{mm}$ saturated soil layer within the frame that is around $8000 \mathrm{~N}$.

A series of shaking table tests have been conducted on the sand specimen in the biaxial laminar shear box since August 2002. Various one- and two-dimensional input motions were imposed by the shaking table. The input motions included sinusoidal $(2$ and $4 \mathrm{~Hz})$ accelerations, with amplitudes $\left(A_{\max }\right)$ from 0.03 to $0.15 \mathrm{~g}$ in the $\mathrm{X}$ or $\mathrm{Y}$ direction, or both. In the two-dimensional shaking, there is a $90^{\circ}$ phase difference between the input acceleration in both $\mathrm{X}$ and $\mathrm{Y}$ directions, i.e., a circular or ellipse motion was applied. The acceleration, with full and reduced amplitudes (recorded at Yuan-Lin and She-Tou seismograph stations in Chi-Chi Earthquake), was also imposed in $\mathrm{X}$ and $\mathrm{Y}$ directions. For example, Table 2 shows the 
TABLE 2-Input motions and settlements in the shaking tests, January 2003.

\begin{tabular}{|c|c|c|c|c|c|c|c|c|}
\hline \multirow[b]{2}{*}{ Shake No. } & \multicolumn{2}{|c|}{$\mathrm{X}$ direction } & \multicolumn{2}{|c|}{ Y direction } & \multirow[b]{2}{*}{$\begin{array}{c}\text { Duration } \\
s\end{array}$} & \multirow{2}{*}{$\begin{array}{l}\text { Height of Sand } \\
\text { After Shaking } \\
(\mathrm{m})\end{array}$} & \multirow{2}{*}{$\begin{array}{c}\text { Dry Density } \\
\text { After Shaking } \\
\left(\mathrm{kg} / \mathrm{m}^{3}\right)\end{array}$} & \multirow[b]{2}{*}{$\begin{array}{l}\text { Dr } \\
(\%)\end{array}$} \\
\hline & $\begin{array}{c}\text { Frequency } \\
(\mathrm{Hz})\end{array}$ & $\begin{array}{c}A_{\max } \\
(\mathrm{g})\end{array}$ & $\begin{array}{c}\text { Frequency } \\
(\mathrm{Hz})\end{array}$ & $\begin{array}{c}A_{\max } \\
(\mathrm{g})\end{array}$ & & & & \\
\hline Pre-shaking & & & & & & 1.393 & 1411 & 11.1 \\
\hline 1 (1-D) & & & 2 & 0.03 & 10 & 1.393 & 1411 & 11.1 \\
\hline $2(1-D)$ & & & 2 & 0.05 & 10 & 1.381 & 1424 & 16.8 \\
\hline $3(1-D)$ & & & 2 & 0.075 & 10 & 1.355 & 1451 & 28.4 \\
\hline $4(1-D)$ & & & 2 & 0.1 & 10 & 1.333 & 1476 & 38.5 \\
\hline $5(2-D)$ & 2 & 0.05 & 2 & 0.05 & 10 & 1.316 & 1495 & 46.3 \\
\hline $6(2-D)$ & 2 & 0.075 & 2 & 0.075 & 10 & 1.299 & 1515 & 54.1 \\
\hline $7(2-\mathrm{D})$ & 2 & 0.1 & 2 & 0.1 & 10 & 1.281 & 1537 & 62.3 \\
\hline $8(2-D)$ & 2 & 0.1 & 4 & 0.1 & 10 & 1.268 & 1553 & 68.6 \\
\hline $9(2-\mathrm{D})$ & 2 & 0.15 & 2 & 0.10 & 10 & 1.252 & 1574 & 75.9 \\
\hline $10(2-D)$ & 2 & 0.15 & 2 & 0.15 & 10 & 1.239 & 1590 & 81.6 \\
\hline $11(2-\mathrm{D})$ & \multicolumn{5}{|c|}{ Chi-Chi Earthquake, She-Tou, PGA $=0.20 \mathrm{~g}$} & 1.209 & 1630 & 95.5 \\
\hline
\end{tabular}

test sequence of the shaking table tests conducted in January 2003. The instrumentation installed on the frames and within the sand specimen recorded the displacements, accelerations, and pore water pressure changes during the shaking tests. The performance of this biaxial laminar shear box is evaluated as follows.

\section{Frame Movements}

Figure 9 shows the plan view of the biaxial movements of the inner frames, which contain the soil specimen, at various depths during a two-dimensional shaking test. It indicates that the multidirectional movements of the soil at different depths induced by the shaking can be realized using the biaxial laminar box.

The rotations of the frames during the shaking tests were examined in every test and it was found that there was very little rotation with less than $0.15^{\circ}$ of rotating angle under the largest shaking. Figure 10 shows the displacements of two different locations on the same inner and outer frames under a two-dimensional horizontal shaking. The movements of the right and left sides of the same frame in both $\mathrm{X}$ and $\mathrm{Y}$ directions are essentially identical, i.e., little rotation of these frames occurred during the test as required by the design.

The consistency of measurements of the frame movements by the accelerometers and LDT was also checked by comparing the double differential of the displacements measured by LDT (with a $10-\mathrm{Hz}$ filter) and the accelerations measured on the same frame. Figure 11 shows one of the comparisons on frame No. 9 in the Janu-

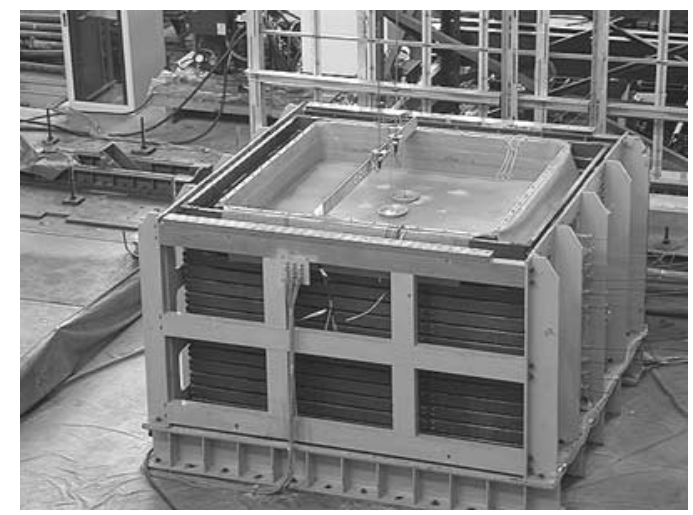

FIG. 15-Laminar shear box on the shaking table. ary 2003 shaking test. It depicts that both measurements gave consistent results of the frame movements. Therefore, the measurements by these sensors are compatible and can be used together for the analyses of soil responses under shaking.

\section{Soil Responses}

Accelerations measured by the accelerometers within the soil and those on the frames during the shaking tests are compared in order to evaluate the consistency between these two measurements. The acceleration time histories within the soil and those of the inner frame at the same depth $(\approx 0.80 \mathrm{~m}$ below the sand surface $)$ and pore water pressure changes near the accelerometer within the soil during a Y-direction sinusoidal shaking with amplitudes of $A_{\max }$ $=0.05 \mathrm{~g}$ and $A_{\max }=0.075 \mathrm{~g}$ are shown in Figs. 12 and 13, respectively. Figure 12 indicates that the induced acceleration within the soil and that of the frame were identical when no liquefaction occurred under the shaking of $A_{\max }=0.05 \mathrm{~g}$. Figure 13 shows that the induced acceleration within the soil and that of the frame were the same before liquefaction, whereas the accelerations measured within the soil decreased substantially after liquefaction while those on the frame became irregular with spikes. Such behavior might be due to the sudden loss of stiffness of the soil when liquefaction occurred. These results indicate that we can use the measured accelerations either within or on the frames of the laminar box to analyze the induced motions during the shaking tests prior to liquefaction. It is not certain whether the measured movements can

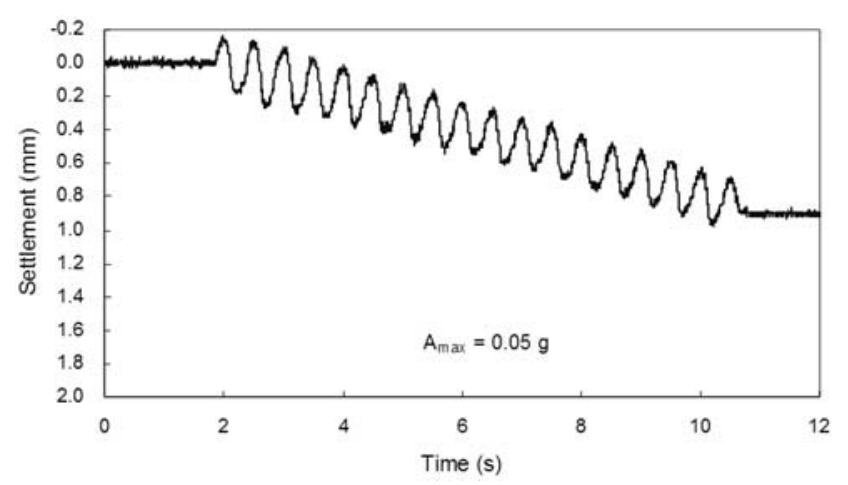

FIG. 16-Settlement measurements during a shaking test. 
represent the real motions of the soil after liquefaction because the sensors might be decoupled from the soil at this stage.

\section{Pore Water Pressure Changes}

The water pressure reading of each piezometer was taken before every shaking test to verify the depth of the sensor for later calculations. A comparison of pore water pressure changes at two different depths in the sand specimen during one- and two-dimensional sinusoidal shakings of the same maximum accelerations is given in Fig. 14. The results show that the pore water pressure generation during a two-dimensional shaking, even with a slightly higher sand density, is higher than that during a one-dimensional shaking. The general trends of pore water pressure changes are similar to those obtained in the shaking tests by others, e.g., Van Laak et al. (1994).

\section{Settlements and Density Changes of the Specimen}

The sand surface settled, i.e., the density of sand increased during each shaking and the amounts of settlement increased with the intensity of shaking as expected, especially when there was liquefaction of the sand. The height of the sand surface after each shaking was measured manually at 16 different locations and the settlement of the sand surface can be calculated. Table 2 also shows the average dry density after each shaking test calculated according to the height of the sand specimen in the shaking tests, January 2003. The surface settlements at the time of shakings can be observed using a settlement plate connected with the magnetostriction type LDT. The laminar shear box containing the sand specimen with the settlement plates is shown in Fig. 15. Figure 16 is an example of the settlement measurements during a shaking test without liquefaction of the sand. Soil samples were also taken using short thinwalled tubes at different depths after the completion of a series of shaking tests and the densities of these samples were obtained.

\section{Conclusions}

A large biaxial laminar shear box was developed for shaking table tests on saturated sand under one- and two-dimensional shakings. The shear box is composed of 15 layers of aluminum alloy frames with a specimen size of 1880 by 1880 by $1520 \mathrm{~mm}$. The soil inside the frame at every depth of the laminar box can have a multidirectional movement, without torsion, in the horizontal plane during a shaking test. A specially designed pluviator was used to prepare the sand specimen inside the shear box by the wet sedimentation method. The uniformity and saturation of the specimen were evaluated and found satisfactory according to the thin-walled tube sampling and P-wave velocity measurements. The shaking table tests of the laminar shear box with or without the sand specimen demonstrated that the performance of the biaxial laminar box fulfills the design requirements. The instrumentation installed within the soil and on the frames could also obtain reliable measurements of the soil movements and pore water pressures during shaking tests. Analyses of some of the shaking table test results have been presented elsewhere (e.g., Ueng et al. 2004) and further tests are underway to obtain more databases for theoretical and numerical analyses of ground responses, liquefaction, and soil-structure interaction under earthquake shakings.

\section{Acknowledgments}

This study was supported by National Science Council, Taiwan, ROC, Grant No. NSC91-2711-3-319-200-03. The technical support in design and manufacturing of the laminar box by the engineers at Mechanical Industry Research Laboratories at Industrial Technology Research Institute, Hsinchu, Taiwan, is gratefully acknowledged. The authors thank H. W. Cheng, C. L. Hwang, W. C. $\mathrm{Ho}, \mathrm{W}$. C. Lee and the engineers at NCREE for their assistance in conducting tests on the shaking table.

\section{References}

De Alba, P., Seed, H. B., and Chan, C. K., 1976, "Sand Liquefaction in Large-Scale Simple Shear Tests," Journal of the Geotechnical Engineering Division, ASCE, Vol. 102, No. 9, pp. 909-927.

Endo O., and Komanobe, K., 1995, "Single- and Multi-directional Shaking Table Tests of Sand Liquefaction," Proceedings, ISTokyo'95, First International Conference on Earthquake Geotechnical Engineering, Tokyo, pp. 675-680.

Fretti, C., Lo Presti, C. F., and Pedroni, S., 1995, “A Pluvial Deposition Method to Reconstitute Well-Graded Sand Specimens," Geotech. Test. J., Vol. 18, No. 2, pp. 292-298.

Hushmand, B., Scott, R. F., and Crouse, C. B., 1988, "Centrifuge Liquefaction Tests in a Laminar Box," Geotechnique, Vol. 38, No. 2, pp. 253-262.

Ishihara, K. and Nagase, H., 1988, "Multi-directional Irregular Loading Tests on Sand," Soil Dyn. Earthquake Eng., Vol. 7, No. 4, pp. 201-212.

Ishihara, K. and Yamazaki, F., 1980, "Cyclic Simple Shear Tests on Saturated Sand in Multi-directional Loading," Soils and Foundations, Vol. 20, No. 1, pp. 45-59.

Kammerer, A. M., Pestana, J. M., and Seed, R. B., 2002, “Undrained Response of Monterey 0/30 Sand Under Multidirectional Cyclic Simple Shear Loading Conditions," Geotechnical Engineering Report No. UCB/GT/02-01, Dept. of Civil and Environmental Engineering, University of California, Berkeley.

Pyke, R. M., Seed, H. B., and Chan, C. K., 1975, "Settlement of Sands Under Multi-directional Loading," Journal of Geotechnical Engineering Division, ASCE, Vol. 101, No. 4, pp. 379-398.

Rad, N. S. and Tumay, M. T., 1987, "Factors Affecting Sand Specimen Preparation by Raining," Geotech. Test. J., Vol. 10, No. 1, pp. 31-37.

Taylor, C. A., Dar, A. R., and Crewe, A. J., 1995, "Shaking Table Modelling of Seismic Geotechnical Problems," Proceedings of 10th European Conference on Earthquake Engineering, Duma, pp. $441-446$.

Ueng, T. S., Chen, C. H., and Chen, H. W., 2004, "Pore Water Pressure Changes in Saturated Sand During One- and Twodimensional Shaking Table Tests," Proceedings of the 11th International Conference on Soil Dynamics \& Earthquake Engineering and the 3rd International Conference on Earthquake Geotechnical Engineering, Berkeley, CA, Vol. 1, pp. 682-687.

Van Laak, P. A., Taboada, V. M., Dobry, R., and Elgamal, A. W., 1994, "Earthquake Centrifuge Modeling Using a Laminar Box," Dynamic Geotechnical Testing II, ASTM STP 1213, R. J. Ebelhar, V. P. Drnevich, and B. L. Kutter, Editors, ASTM International, West Conshohocken, PA, pp. 370-384. 